\title{
A Tutoria como Estratégia Educacional no Ensino Médico
}

\section{Mentoring as an Educational Approach in Medical Education}

\author{
Leandro Jerez Chaves ${ }^{I}$ \\ Eduardo César Queiroz Gonçalves ${ }^{I}$ \\ Larissa Ramos Ladeiral \\ Mário Sérgio Ribeiro ${ }^{I}$ \\ Mônica Barros Costa \\ Andréia Aparecida de Miranda Ramos ${ }^{I}$
}

\section{PALAVRAS-CHAVE \\ - Preceptoria; \\ - Educação Médica; \\ - Mentores; \\ - Orientação.}

\section{KEYWORDS}

- Preceptorship;

- Medical Education;

- Mentors;

- Orientation
Reencaminhado em: 16/07/2013

Reencaminhado em: 10/12/2013

Aprovado em: 31/08/2014

The mentoring relationship dates back to prior to the invention of written language. There have been changes in its structure, but it has not lost the essence of promoting the development of the apprentice. Mentoring is a personalized learning process, in a dynamic relationship, which has great importance as a learning instrument in undergraduate courses and professional training. In medical education, it is positively related to scholarly support, career satisfaction, academic production and a reduced risk of burn-out. However, it is still limited by the small number of trained mentors, participants' lack of time and lack of institutional support. This paper reviews the concepts of mentoring, the attributes of mentors and apprentices and their relationship, and assesses the importance, the expectations and the limitations of mentoring in medical education, especially in Brazilian public medical schools. A review of scientific articles on the PubMed database and published between 2005 and 2011 was performed, as well as other publications cited in the selected articles. 


\section{HISTÓRICO}

O desenvolvimento da linguagem escrita permitiu que o modelo de ensino em saúde, utilizado na Antiguidade por curandeiros e feiticeiros, perdesse seu caráter "estacionário e imitativo" e adquirisse maior formalidade. Na Grécia, a concepção de cultura e do lugar do indivíduo na sociedade cresceu concomitante ao surgimento das polis. Entende-se por polis não simplesmente a organização das cidades-estado dos gregos, mas uma reorganização política e cultural, que, apesar de diferir em cada localidade, tinha características comuns: o poder se transferiu da força bruta para o domínio da palavra; os deuses perderam o centro do poder para as leis; e as manifestações importantes da vida social eram públicas e ocorriam principalmente nas ágoras, praças onde existiam os mercados e as assembleias do povo, possibilitando o desenvolvimento intelectual e político graças a discussões, argumentações e polêmicas. A polis de Atenas foi a que mais se desenvolveu, tornando-se o centro intelectual da Grécia, fundado na liberdade de expressão e na condução coletiva dos negócios públicos ${ }^{1}$. A liberdade de expressão foi responsável por atrair matemáticos, arquitetos, artistas, pensadores e sofistas, também conhecidos como professores da oratória, para o local. Estes professores eram contratados pelos membros da oligarquia ateniense e estabeleciam uma relação de tutor e aprendiz baseada em estima mútua, afetividade e amizade. A relação não era limitada a uma área de conhecimento, mas abrangia ensinamentos éticos e morais. Já na cultura romana, a formação era pragmática e envolvia tanto a família quanto a escola, sendo considerada primordial a educação provida pela família² .

Durante a teocêntrica Idade Média, a educação ocorria em escolas, junto aos mosteiros, visando interpretar os textos sagrados, combater a heresia e converter os infiéis. Era tida como um instrumento de salvação da alma e obtenção da vida eterna. Com a ascensão burguesa, no final desse período, foi necessária uma mudança no sistema educacional, surgindo as escolas seculares, que possibilitaram à nova classe o aprendizado de leitura, escrita e cálculo ${ }^{3}$.

As universidades foram fundadas no início do século XIII por associações de estudantes e professores ${ }^{4}$. Na primeira delas, a Universidade de Nápoles, o aluno era designado a um professor, que ficava responsável por the ensinar habilidades específicas, como a leitura. Este aluno, supervisionado pelos mestres, passava então a ensinar os estudantes mais jovens. Essa relação define claramente a existência da relação tutor-aprendiz nos primórdios daqueles novos centros educacionais ${ }^{5}$.

Entre os séculos XVI e XVIII, as universidades perderam a autonomia do ensino e, em consequência, o prestígio, ao passarem ao controle de poderes políticos (Charle, 1996 apud Geib et al. $\left.{ }^{2}\right)$. Tal cenário impulsionou o sistema de tutoria, reforçando a individualidade do processo ensino-aprendizagem, uma vez que os alunos migravam das universidades para colégios nos quais havia atividades como internato, teatro e tutoria. Já no século XIX, com o advento da Revolução Francesa e da Revolução Industrial, as universidades recuperaram sua importância, e o ensino tornou-se oficialmente laico e aberto às mulheres. Foram criadas as faculdades tradicionais, como Direito, Medicina, Teologia, Ciências e Letras. No século seguinte, consolidaram-se os distintos modelos de universidade (francês, alemão, inglês e norte-americano) concomitantemente à expansão mundial das mesmas. Com isso, a tutoria passou a ser vista de forma diferente e empregada ou não de acordo com cada um desses modelos ${ }^{2}$.

\section{OBJETIVO}

O presente artigo foi elaborado com objetivo de avaliar a importância da tutoria na educação médica, através da revisão de artigos científicos do período 2005-2011, no banco de dados PubMed, assim como do uso de referências citadas nos artigos selecionados.

Inicialmente, foram abordados, de forma sucinta, os principais conceitos de tutoria, seguidos dos tópicos sobre a tutoria na educação médica, sua importância, limitações e expectativas, e o contexto e perspectivas da tutoria nas escolas médicas do Brasil.

\section{O QUE É TUTORIA?}

Para melhor compreender o que é a tutoria, é necessário entender os conceitos de mentor, tutor e preceptor. Botti e Rego ${ }^{6}$ analisaram os conceitos e como esses termos são utilizados na literatura médica e definiram o mentor como um profissional mais experiente que auxilia o aprendiz ao longo do seu crescimento pessoal e profissional. Interessa-se e investe no desenvolvimento dos menos experientes, tentando estabelecer uma relação mais próxima e mais pessoal. Não desempenha o papel de avaliador e influi na formação da personalidade do aprendiz, por meio do estímulo à capacidade crítica e de reflexão. $\mathrm{O}$ tutor é definido como o profissional cuja função é ensinar o aluno a aprender, tendo seu conceito articulado ao campo semântico de proteção. É novamente um profissional experiente que aconselha, facilita a construção do conhecimento, atua como guia e modelo, principalmente no campo profissional. Pode aconselhar e oferecer suporte no âmbito pessoal, apesar de não ser sua principal função. Já o termo preceptor, na literatura médica, é utilizado para aqueles que dão suporte, orientam, ensinam, compartilham experiências, melhoram as competências clínicas do aprendiz e o ajudam 
a se adaptar ao exercício da profissão. Conhecimento e habilidade clínica são atributos essenciais para desempenhar a essência de sua tarefa: ensinar a clinicar. O foco primordial é no desenvolvimento profissional, entretanto podem servir de modelo para o desenvolvimento pessoal e auxiliar a formação ética, realizando funções típicas dos mentores. Segundo os mesmos autores, em nosso meio, o termo "tutor" pode ser utilizado em substituição ao termo "mentor", designando a atividade exercida por este. Isto ocorre devido à inexistência de tradução para a língua portuguesa dos termos mentorship e mentoring. Neste artigo, utilizaremos o termo tutor seguindo esta afirmativa ${ }^{6}$.

Assim sendo, neste artigo abordaremos a tutoria como uma forma especial de serviço educacional individualizado. É uma relação dinâmica, que envolve tempo integral, durante a qual ambas as partes definem e redefinem suas funções. Deve ser sempre considerada um processo, nunca um resultado ${ }^{7,8}$.

Tem como função promover o desenvolvimento profissional e o amadurecimento pessoal do aprendiz, por meio da abordagem de temas amplos ${ }^{5}$. Quando utilizada como ferramenta de ensino, em graduações e treinamentos profissionais, aborda temas relacionados ou não à grade curricular?

O sucesso da relação tutor-aprendiz e, consequentemente, do programa de tutoria requer ativa participação de ambas as partes, num ambiente de igualdade, liberdade e responsabilidade $^{7,8,9}$.

\section{TIPOS DE TUTORIA}

Não há um consenso ou uma classificação padrão dos tipos de tutoria. Os diversos modelos de relação entre tutor e aprendiz podem ser analisados sob vários aspectos. Frei e colegas ${ }^{7}$, em sua revisão sistemática, utilizam a classificação de tutoria de grupos, tutoria de pequenos grupos, tutoria um-para-um, peer-mentoring e tutorias que misturam os modelos citados (de grupo, tutoria um-para-um e tutoria de pequeno grupo $)^{7,10}$.

Entende-se por tutoria de grupo aquela na qual há um tutor, que seja um profissional formado, preparado de forma adequada para esta tarefa e que não necessariamente ocupe uma posição de destaque na carreira, e aprendizes com perfil profissional semelhante. A tutoria de pequeno grupo envolve geralmente até oito aprendizes, e a de grupos envolve maior número de pessoas. Trata-se de um modelo muito utilizado nas graduações e está destinado a ter melhores resultados quando os envolvidos já se conhecem e o grupo se torna coeso, facilitando transpor barreiras, como a organização dos encontros e a confidencialidade do grupo. Os pontos positivos desta modalidade são a troca de experiências profissionais e pessoais, e o encorajamento mútuo ${ }^{10}$.
$\mathrm{Na}$ tutoria um-para-um, também conhecida como $d y a-$ dic mentoring, o processo é extremamente individualizado e avança concomitantemente ao desenvolvimento profissional do aprendiz. Este modelo exige que o tutor, além de adequada formação, possua uma posição profissional de destaque. Trata-se do modelo mais adequado para aqueles que já estão avançados na formação profissional, como ocorre nos processos de pós-doutorado ${ }^{10}$.

O peer-mentoring é o modelo de tutoria no qual os aprendizes são alunos no início da formação profissional, e os tutores são alunos mais avançados no processo de formação. São estabelecidos pares dentro do grupo, e metas são traçadas para as duplas e o grupo. Trata-se de um modelo com menor disparidade de poder, permitindo o compartilhamento de opiniões, planos pessoais, problemas do dia a dia, oportunidade de reflexão, além de apoio pessoal e social ${ }^{10}$.

A classificação que define a relação tutorial como formal e informal é a mais utilizada nas publicações cientificas sobre tutoria na educação médica $7,9,11,12$.

A tutoria informal ocorre por acaso, quando dois indivíduos são unidos por interesses comuns, resultando numa relação entre "mestre e aprendiz" espontânea ou acidental. Esse tipo de aprendizado encontra-se descrito em vários momentos da história, como ocorreu entre Athena e Telemachus (na Odisseia de Homero), Sócrates e Platão, Freud e Carl Jung, Carlos Chagas e Oswaldo Cruz, dentre outros. É caracterizada por um relacionamento mutuamente satisfatório, de longo prazo, que não segue a estrutura de nenhuma instituição ${ }^{11}$.

A tutoria formal, por outro lado, é planejada e apoiada institucionalmente. Envolve a atribuição do(s) aprendiz(es) ao(s) tutor(es) e a tentativa de construir o tipo e a qualidade da relação observada na tutoria informal. Nem sempre este objetivo é alcançado e por isso a tutoria formal não é obrigatoriamente efetiva, estando sujeita a relações de disparidade de poder. Trata-se de um processo com reconhecida importância para a pesquisa e percepção de suporte acadêmico, constituindo uma tentativa de oferecer à maioria dos alunos a experiência que a minoria consegue através da tutoria informal ${ }^{11}$.

\section{CARACTERÍSTICAS DA RELAÇÃO DE TUTORIA}

Segundo Jackson et al. ${ }^{13}$, para que ocorra uma relação mais dinâmica e recíproca na tutoria, é necessária a existência da "the right chemistry" (p.329), o que acreditamos tratar-se principalmente de empatia, que pode ser definida como a "forma de identificação intelectual ou afetiva de um sujeito com uma pessoa, uma ideia ou uma coisa"14 (sem local). Como a tutoria deve ser focada no aprendiz, este deve buscar o tutor com quem tenha maior compatibilidade, para que a experiência lhe 
seja proveitosa ${ }^{15}$. Uma vez que o processo tutorial é, na essência, uma via de mão dupla, tanto aprendizes quanto tutores apresentam crescimento profissional e pessoal ${ }^{11,15,16}$.

Diversos aspectos da tutoria estão ligados à relação que é desenvolvida entre os participantes e aos seus comportamentos. O tutor deve prover segurança emocional e suporte e não deve fazer julgamentos ou censuras, mas apoiar o aprendiz de forma empática em suas dificuldades. Cabe ao tutor estimular a independência do aprendiz, induzindo a reflexão e síntese sobre os conhecimentos e habilidades adquiridos, para que este possa ter papel mais ativo durante as práticas ${ }^{15}$. $\mathrm{O}$ respeito é fundamental, mesmo que a informalidade no tratamento seja uma característica positiva para o estabelecimento da parceria.

A existência de uma disparidade de poder, ocupando o tutor uma posição superior à do aprendiz, é identificada nos programas de tutoria formal que geralmente se estruturam ao redor de um tutor que seja um profissional experiente e respeitado. Esta relação de poder acaba por pressionar o aprendiz a cumprir as metas acordadas, pelo temor de desapontar o tutor $^{17}$. No entanto, acreditamos que esta mesma disparidade se encontre nos processos informais, e este esperado envolvimento do aprendiz com o processo de tutoria é apontado como fundamental para a satisfação do tutor na relação ${ }^{16}$.

\section{ATRIBUTOS DO TUTOR E DO APRENDIZ}

O tutor é, de forma geral, um profissional experiente e respeitado. Sua função é semelhante à de um conselheiro, professor ou mesmo guia. O papel de tutor envolve também prover suporte emocional, interessar-se pelas necessidades dos estudantes, compartilhar suas experiências e guiá-los em seu crescimento tanto individual quanto social ${ }^{18}$.

O tutor deve ser capaz de instigar reflexões e acompanhar o aprendiz na execução das metas traçadas. Para isso, pode formular perguntas que gerem respostas discursivas; evitar propor soluções para problemas que surgem, estimulando os aprendizes a reconhecê-los e a proporem maneiras de alterá- $\operatorname{los}^{19}$; encorajar o aprendiz a acreditar nas próprias ideias, promovendo críticas úteis e construtivas ${ }^{8}$. Deve ter aptidão para a comunicação, apresentar características pessoais como paciência, tolerância, senso ético, autenticidade e controle emocional ${ }^{2}$

Outra característica importante do tutor é respeitar a singularidade, reconhecer as contribuições e compartilhar os resultados alcançados com os aprendizes ${ }^{12}$. Ao ser incapaz de identificar os objetivos e valores do aprendiz, o tutor pode distorcer o processo tutorial, podendo inclusive comprometer o desenvolvimento da identidade profissional do aprendiz ${ }^{11}$.
Como a relação de tutoria é uma "via de mão dupla", na relação funcional o tutor também vivencia mudanças - como professor, ao conhecer e se aproximar mais dos estudantes e de suas ideias; como membro da academia, ao perceber a instituição como um todo e aprimorar seu currículo; e também como pessoa, ao repensar suas relações, valores e autojulgamentos. Apesar de todos estes aspectos, poucos estudos avaliaram a percepção dos tutores ${ }^{16,20-23}$.

Bellodi ${ }^{16}$ explorou a percepção dos 80 tutores da Faculdade de Medicina da Universidade de São Paulo (FMUSP) a respeito da experiência de tutoria formal em grupos dos quais participam. Eles relataram como situações que os fizeram se sentir verdadeiramente como tutores aquelas em que podiam compartilhar suas experiências, promover bom aconselhamento, ajudando na tomada de decisões, e quando eram procurados pelo aprendiz em momentos não agendados ${ }^{16,23}$.

O aprendiz geralmente é uma pessoa mais nova do que seu tutor, em busca de auxílio e orientação. Deve ter um papel ativo no desenvolvimento da relação, uma vez que esta é centrada nele e tem como objetivo beneficiá- $\mathrm{lo}^{20}$. A adesão e o envolvimento do aluno são apontados como fundamentais para o estabelecimento da dinâmica relação de tutoria e da satisfação do tutor ${ }^{16}$. Nos programas formais, é fundamental que o aluno se envolva com a tutoria e não julgue esta atividade menos importante para seu currículo em relação a outras, como ligas acadêmicas, iniciação científica e estágios ${ }^{21,23}$.

Antes de um aprendiz se envolver com a tutoria, é necessário que haja uma etapa introspectiva na qual ele defina claramente seus valores, o estilo individual de trabalho e deficiências educacionais. Em concordância com estes aspectos, metas de curto, médio e longo prazos poderão ser traçadas e deverão ser observadas e redefinidas periodicamente. Desta maneira, espera-se que o aprendiz seja mais responsável por seu crescimento e possa tornar a tutoria mais efetiva ${ }^{23,24}$.

Os aprendizes esperam de seus tutores generosidade para compartilhar suas experiências, disponibilidade para manter uma relação de longo prazo, credibilidade, confidencialidade, habilidades de comunicação, liderança e senso de humor. Esperam com a relação se tornar mais conscientes de seus limites e potenciais, bem como aumentar a autoconfiança no presente e no futuro pessoal e profissional ${ }^{23}$.

Alguns estudos avaliaram as dimensões da relação tutorial na perspectiva dos aprendizes e identificaram quatro pontos relevantes para a relação tutorial: exploração pessoal com reconhecimentos dos valores individuais, orientação prática para crescimento profissional e pessoal, apoio do tutor e o conselho dos tutores para o desenvolvimento de habilidades ${ }^{13,25,26}$. 


\section{IMPORTÂNCIA, EXPECTATIVAS E LIMITAÇÕES DA TUTORIA NA EDUCAÇÃO MÉDICA}

Os programas de tutoria representavam uma ferramenta comumente utilizada nas escolas de Enfermagem e foram introduzidos na educação médica nos Estados Unidos e Inglaterra na década de 1990, servindo, a princípio, de suporte para as mulheres e minorias étnicas dentro da carreira médica ${ }^{7,27}$.

Diversos estudos sobre a tutoria na educação médica demonstraram a sua contribuição para o desenvolvimento pessoal e profissional dos aprendizes ${ }^{11,17,28,29}$, principalmente nos seguintes aspectos: satisfação com a carreira ${ }^{9,11,21,28}$, pesquisa e produção científica ${ }^{17,28}$, percepção de suporte acadêmico e profissional ${ }^{12,17,21,28}$, redução do risco para síndrome de burn-out ${ }^{11,28}$ e desenvolvimento de relações dentro da profissão ${ }^{28,29}$.

Em relação aos tutores, o processo de tutoria é relevante em três aspectos, considerados motivadores pelos profissionais. Como professores, passam a entender melhor as necessidades e características dos alunos, desenvolvendo relações mais próximas. Como membros da academia, percebem a universidade como um todo, não se limitando apenas à sua área de atuação, além de obter melhoria na qualidade de seu currículo. Como pessoas, têm suas relações, valores e autojulgamentos revisados ${ }^{16}$.

Embora os estudos sobre tutoria na área médica tenham aumentado nas duas últimas décadas, eles ainda são limitados. Apresentam grande variedade no conceito de tutoria e se preocupam mais em mostrar os resultados que os aprendizes podem alcançar ${ }^{11}$, principalmente no que diz respeito à produção acadêmica, do que com o processo que ocorre durante todo o período da relação tutor-aprendiz ou mesmo com a percepção dos tutores sobre esta ferramenta ${ }^{7,11,16,23,30}$.

Na prática, o grande número de alunos nas faculdades de Medicina em todo o mundo dificulta a disponibilidade dos programas de tutoria. Uma estratégia possível é a tutoria em pequenos grupos, abrangendo até oito aprendizes, o que permite maior facilidade para a confluência de horários e menor demanda de tutores ${ }^{7,17}$.

Pololi e Knight ${ }^{11}$ avaliaram a tutoria dentro da carreira acadêmica médica nos Estados Unidos. Seu trabalho comparou o método de tutoria tradicional, no qual a relação era desenvolvida individualmente, denominada Personal Mentoring Program, e a tutoria em grupo, do tipo peer-mentoring, no programa denominado Collaborative Monitoring Program. Segundo os autores, a tutoria em grupo do tipo peer-mentoring é superior ao método tradicional, porque permite desenvolver uma relação forte e confiável entre os membros do grupo, impede o uso inadequado de poder e compensa a falta de tutores ${ }^{11}$.
Um exemplo bem-sucedido de peer-mentoring é o realizado pelo grupo Internal Medicine Research Group at Emory (IMeRGE), composto pelo corpo discente júnior da Universidade de Emory, em Atlanta. Bussey-Jones et al. ${ }^{17}$ relatam que neste grupo os discentes recém-ingressos são pareados por idade, experiência e classificação na faculdade, o que permite uma relação mais flexível e mútua. O grupo possui um consultor sênior, com função semelhante à de um tutor. O objetivo do grupo é fazer com que mais estudantes de Medicina e médicos residentes escolham e permaneçam na carreira acadêmica. Segundo os autores, o desenvolvimento deste novo método ocorreu devido às limitações da tutoria tradicional, a tutoria de um-para-um, para estes aprendizes, principalmente pela indisponibilidade de tutores. Outro problema identificado foi a homogeneidade criada quando o aprendiz se torna semelhante ao seu tutor num círculo vicioso, no qual os avanços inovadores podem ser limitados, além do potencial risco de exploração decorrente da disparidade de poder da qual o "tutor" possa se aproveitar. Os autores reconhecem ainda, como limitações do peer-mentoring, a competição entre os pares de estudantes e/ou entre as duplas, a menor troca de experiência profissional em comparação com a tutoria tradicional e a falta de avaliação da sua efetividade ${ }^{17}$.

Outra limitação da tutoria no aprendizado médico, citada por tutores e aprendizes, é a falta de tempo. Devido à extensa carga horária e grande número de atividades extracurriculares, nem sempre é possível realizar encontros frequentes. Essa falta de tempo pode levar ao desligamento de tutores, agendamento irregular dos encontros, comunicação não efetiva e, consequentemente, menor envolvimento do aprendiz ${ }^{16,21}$. Por outro lado, a tutoria poderia se desenvolver durante um período mais prolongado, dado o tempo de duração dos programas de graduação e pós-graduação ${ }^{11,30}$.

Outros obstáculos se relacionam a fatores organizacionais do meio acadêmico: os docentes são avaliados prioritariamente pela produção científica em detrimento do envolvimento com o ensino; quanto ao aluno, há uma falta de recompensa e reconhecimento público pelos professores participantes, além da competição entre os discentes ${ }^{23}$.

Na experiência brasileira, a falta de interesse e informação ou falha da percepção da necessidade de participar do programa por parte dos discentes são limitações relatadas em publicações sobre programas de tutoria em grupo. Um dos motivos da baixa adesão pode ser o estabelecimento de relações de tutoria informal que, na concepção do discente, supriria a necessidade da experiência formal ${ }^{21,30}$.

Além disso, tutores e aprendizes podem ter diferentes metas, comprometimentos, demandas e expectativas com o 
processo de tutoria ${ }^{11}$. Para os tutores, pode ser difícil entender a relutância dos alunos em participar dos programas e, consequentemente, encontrar maneiras de incentivar sua adesão. Isso pode diminuir a satisfação do tutor e pôr em risco a relação de tutoria ${ }^{16}$.

No que diz respeito à avaliação dos programas de tutoria, o maior problema é o fato de não ser baseada em questionários validados, sendo as ferramentas disponíveis desenhadas para cada programa específico, dificultando comparações, o que torna incipiente a comprovação da importância da tutoria for$\mathrm{mal}^{7,12}$. Os pontos avaliados nestes estudos geralmente dizem respeito ao programa e aos encontros - experiências, frequências e regularidade dos encontros, temas abordados, confronto de realidade e expectativas da relação e satisfação dos envolvidos -, ao tutor e ao aprendiz - atributos, habilidades e competências adquiridas ${ }^{23,31}$. Apesar do consenso de que os programas devem ser avaliados periodicamente, alguns estudos citam a escassez de relatos sobre a sua efetividade ${ }^{7,27}$, enquanto outros tentaram medir esta variável e identificaram aspectos que devem ser considerados nas avaliações e na elaboração dos programas ${ }^{12,13,25,26,32}$. Outras limitações dos processos de avaliação são a dificuldade de isolar a influência do programa de tutoria, uma vez que não é a única fonte de suporte do aprendiz, e a escassez de estudos sobre a percepção dos tutores em relação ao processo e suas dificuldades dentro da relação tutorial ${ }^{7,16}$.

Um estudo relevante sobre o processo tutorial na residência médica foi o conduzido por Ramanan et al..$^{28} \mathrm{com}$ o intuito de identificar as atividades de tutoria e a relação entre tutoria e preparo profissional entre os residentes de cinco hospitais afiliados da Faculdade de Medicina de Harvard. Noventa e três por cento dos residentes consideraram importante ter um tutor durante a residência médica, mas cerca da metade deles não teve. $\mathrm{O}$ estudo mostrou ainda que apenas metade dos residentes participava de um programa formal de tutoria. Os residentes não procuraram um tutor por medo de se aproximar de um membro da faculdade, por não encontrar alguém em quem confiassem ou por nunca terem pensado em se aproximar de um orientador com este objetivo. Todavia, residentes que participavam de programas tutoriais descreveram a experiência como positiva para o desenvolvimento profissional, fato corroborado pela expressiva porcentagem que afirmou ter recebido conselhos úteis sobre a carreira e o exercício da profissão. Por outro lado, apenas um quarto dos indivíduos avaliados afirmou que recebeu assistência quando enfrentou problemas pessoais, e a relação pessoal com o tutor não foi uma variável estatisticamente associada à satisfação com o programa tutorial, achados que sugerem que nestas relações de tutoria o desenvolvimento pessoal não é priorizado ${ }^{28}$.

\section{CONTEXTO E PERSPECTIVAS DA TUTORIA NAS ESCOLAS MÉDICAS DO BRASIL}

O estudo desenvolvido por Silva et al. ${ }^{33}$ mostrou que cerca de $32 \%$ das escolas médicas brasileiras possuem infraestrutura para o desenvolvimento do processo de ensino-aprendizagem e oferecem tutorias. Além das limitações estruturais presentes na maioria das escolas médicas, outros fatores - como a falta de qualificação de tutores e o não reconhecimento formal dos programas - dificultam a implantação do processo de tutoria. Apesar deste contexto, existem alguns relatos de experiências em escolas médicas de universidades públicas no Brasil.

De 1990 a 1998, a Faculdade de Medicina de Botucatu (Unesp), em São Paulo, desenvolveu o Programa Professor-Tutor. Tratava-se de um programa de tutoria formal, de adesão voluntária de tutores e aprendizes, que pretendia oferecer uma alternativa de apoio ao estudante. $\mathrm{O}$ programa não oferecia nenhuma creditação ao aluno, concorria com outros compromissos dos professores e não era adequadamente valorizado. Assim, a atividade foi descontinuada em 1998, sem avaliação ${ }^{23}$.

Em 1997, a Faculdade de Medicina de Marília iniciou o Programa de Orientação de Estudantes da Famema. A implantação foi anual e gradual, envolvendo uma nova série a cada ano. Atingiu 100\% dos estudantes em 2002 e se expandiu para a faculdade de Enfermagem em 2004. Trata-se de um programa de caráter obrigatório, no qual o aluno permanece com o mesmo orientador durante toda a graduação $0^{23}$. Uma avaliação do programa mostrou que os estudantes reconheceram sua pertinência e o empenho dos envolvidos e identificou como pontos negativos a falta de interesse e compromisso dos estudantes e a dificuldade relacionada à disponibilidade de tempo, por parte tanto dos orientadores quanto dos orientados ${ }^{34}$.

Em 2000, o colegiado de Medicina da Universidade Federal de Minas Gerais criou o projeto Tutoria. Trata-se de um programa de tutoria em grupo para os alunos que cursam a disciplina Módulos de Saúde B - Tutoria, no quinto período da graduação. Neste programa, o tutor orienta os alunos nas atividades curriculares e optativas, colaborando no desenvolvimento técnico e emocional do aprendiz. O projeto conta com apoio de terapia especializada no Núcleo de Apoio Psicopedagógico aos Estudantes de Medicina ${ }^{35}$.

Também no ano de 2000 foi implantado o projeto Tutor na Faculdade de Medicina de Rio Preto (Famerp). Os tutores deste programa passaram por processo de capacitação. $\mathrm{O}$ tipo de tutoria desenvolvida foi de grupos, com oito a 12 alunos por tutor. O programa foi avaliado principalmente em relação à satisfação dos envolvidos, interesse pela atividade e desejo de continuidade. A maioria dos tutores e alunos se declarou 
satisfeita com o programa, interessada e desejava continuar no programa. O programa se estendeu à Faculdade de Farmácia em $2003^{23}$

O Programa Tutores da Faculdade de Medicina da Universidade de São Paulo, por sua vez, iniciou suas atividades em 2001, no modelo de tutoria em grupo. Os tutores passaram por processo de seleção e capacitação. Cada grupo é formado por alunos de todos os anos da graduação e discute temas relacionados à formação médica, à área científica, ao desenvolvimento pessoal e à cidadania. Há um programa de temas predefinidos de acordo com as expectativas dos estudantes, envolvendo assuntos ligados a ética, relação médico-paciente, responsabilidade social, opções profissionais, ensino e aprendizado, entre outros. O programa é curricular, na modalidade de disciplina optativa. Trata-se de uma ferramenta para integração e troca de experiências entre alunos e professores, e identificação de problemas na graduação, contribuindo para a formação integral do futuro médico. Os tutores são supervisionados, treinados e acompanhados pela organização do programa. Apesar do apoio institucional, o programa tem como limitação a baixa adesão de alunos. Dentre os motivos identificados para explicar a baixa adesão, destacam-se a ocorrência de tutoria informal, a dinâmica ruim de alguns grupos, a preferência por outras atividades acadêmicas, como ligas acadêmicas, estágios e iniciação científica, bem como a não valorização das atividades de tutoria nos processos seletivos para residências médicas ${ }^{21}$.

Outro programa de tutoria formal em grupos existe na Faculdade de Medicina da USP - Ribeirão Preto. Implantado em meados de 2003, tem como objetivo assistir os alunos pelo menos durante o primeiro ano após o ingresso na graduação. O estudo desenvolvido por Colares et al. ${ }^{30}$ descreveu a constituição destes grupos: de seis a oito alunos recém-admitidos, o tutor e dois alunos de outros períodos que o auxiliavam. A seleção dos alunos e tutores foi realizada pela inscrição voluntária para o projeto, havendo um processo de seleção e treinamento. O objetivo é contribuir para a construção da identidade médica dos alunos, facilitar sua adaptação à faculdade, identificar e prevenir dificuldades acadêmicas e emocionais. Após um ano, avaliou-se a percepção dos mentores e aprendizes sobre o programa e foi possível concluir que os alunos participantes e não participantes e os tutores perceberam o programa como positivo, apesar de serem necessários ajustes. Um ponto negativo identificado no estudo foi a falta de interesse, informação ou falha da percepção da necessidade de participar do programa por parte dos discentes ${ }^{30,36}$.

A Faculdade de Medicina da Universidade Federal de Juiz de Fora (FMUFJF) possui experiência na tutoria em grupo. $\mathrm{O}$
Grupo de Educação Tutorial (GET - Medicina) iniciou suas atividades em maio de 2010, composto por três tutores e três acadêmicos, e atualmente conta com seis acadêmicos e um tutor. Dentre seus objetivos, destacam-se o aprendizado tutorial de natureza coletiva e interdisciplinar, melhora da qualidade da graduação, qualificação dos profissionais e dos docentes, formulação de estratégias de desenvolvimento e modernização do ensino, estímulo ao espírito crítico e à conduta ética e cidadã, inerentes à educação superior. Trata-se de um programa longitudinal no qual o aluno, selecionado por meio da avaliação dos conhecimentos sobre educação médica, Atenção Primária em Saúde, doenças crônico-degenerativas, álcool, drogas e língua estrangeira, pode permanecer até a conclusão da graduação. As atividades desenvolvidas são variadas e envolvem acompanhamento de disciplinas integradoras do ciclo básico e profissionalizante, de Grupos de Aperfeiçoamento Profissional do Programa de Educação Permanente do Estado de Minas Gerais, construção de um plano de desenvolvimento pessoal, atividades de pesquisa e extensão, como o acompanhamento de ambulatórios multiprofissionais. Não há avaliação formal do programa, mas a avaliação realizada, nos moldes daquela adotada pelo programa PET do MEC, mostrou que os tutores foram considerados preparados e envolvidos com a atividade. As principais dificuldades apontadas foram falta de tempo devido à extensa carga horária da graduação, necessidade de cumprir outras atividades valorizadas nos processos seletivos de residências médicas e limitações de espaço físico.

\section{CONCLUSÃO}

A relação de tutoria pode ser observada anteriormente à invenção da linguagem escrita. Desde seu surgimento, ocorreram mudanças em sua estrutura, mas sem perda de sua essência de promover o desenvolvimento do aprendiz. Nos dias de hoje, a tutoria é ferramenta de diversas graduações e programas de treinamento profissional. Trata-se de um processo que demanda envolvimento de ambas as partes, sendo de fundamental importância a identificação dos atributos de tutores e aprendizes e as características da relação entre eles.

Na educação médica, a tutoria é importante, sobretudo para aprimorar a produção científica e o suporte acadêmico, reduzir o risco de burn-out e promover o desenvolvimento de relações dentro da profissão e a satisfação com a carreira. Todavia, existem limitações relacionadas ao grande número de alunos e ao pequeno número de tutores preparados, além da falta de tempo dos participantes e de apoio institucional.

A falta de programas formais de tutoria nas graduações e nas residências médicas não ocorre somente nos países em de- 
senvolvimento, e esforços devem ser feitos para transformar esta realidade. É necessário mostrar aos alunos que a tutoria é tão importante para a formação médica quanto as demais atividades acadêmicas tradicionais, como ligas, estágios, projetos de pesquisa e de extensão, dentre outros, que são valorizadas nos processos de seleção de treinamentos profissionais.

A escassez de relatos - e sobretudo de avaliações - de grupos tutoriais das escolas médicas brasileiras provavelmente se deve ao maior número de processos informais ou mesmo pela não divulgação dos projetos formais em execução. $\mathrm{O}$ compartilhamento das experiências e dificuldades entre as instituições pode ser importante no processo de aperfeiçoamento e superação, no qual a criatividade pode contribuir para que maior número de alunos tenha acesso a esta ferramenta.

\section{REFERÊNCIAS}

1. Almeida RA. Panorama histórico da filosofia política, da antiguidade ao período pós-revolucionário. Jus Navigandi [S.I.]; 2005 [capturado 11 jul 2013]. Disponível em: http:/ / jus.com.br/revista / texto/7417 / panorama-historico-da-filosofia-politica-da-antiguidade-ao-periodo-pos-revolucionario.

2. Geib LTC, Krahl M, Poletto DS, Silva CB. A tutoria acadêmica no contexto histórico da educação. Rev. bras. Enferm. 2007; 60(2) [capturado 19 set. 2011]. Disponível em: <http://www.scielo.br/scielo.php?script=sci_ arttext\&pid=S0034-71672007000200017\&lng=en\&nrm=i so>.

3. Aranha MLA. História da educação. São Paulo (SP): Moderna; 1989.

4. Minogue KR. O conceito de universidade. Brasília: Editora UnB; 1977.

5. Monroe P. História da educação. São Paulo (SP): Editora Nacional; 1979.

6. Botti SHO, Rego S. Preceptor, supervisor, tutor e mentor: quais são seus papéis? Rev bras educ méd 2008; 32(3) [capturado 10 dez. 2013]. Disponível em: <http:/ / www.scielo. br/scielo.php?pid=S0100-55022008000300011\&script=sci_ arttext>.

7. Frei E, Stamm M, Buddeberg-Fischer B. Mentoring programs for medical students - a review of the PubMed literature 2000 - 2008. BMC Medical Education 2010;10(32) [capturado 19 set. 2011]. Disponível em: < http:/ /www. biomedcentral.com/1472-6920/10/32/>.

8. Garmel GM. Mentoring medical students in academic emergency medicine. Academic Emergency Medicine 2008;11(12) [capturado 19 set. 2011]. Disponível em: <http://onlinelibrary.wiley.com/doi/10.1197/j.aem.2004.06.013/pdf >.
9. Mclaughlin C. Mentoring: What Is It? How Do We Do It and How Do We Get More Of It? Health Services Research 2010; 45(3) [capturado 19 set. 2011]. Disponível em: < http:/ / onlinelibrary.wiley.com/doi/10.1111/j.1475-6773.2010.01090.x/ pdf $>$.

10. Buddeberg-Fischer B, Stamm M. Mentoring in Medicine: Forms, Concepts and Experiences. State Secretariat for Education and Research SER, Suíça, 2012 [capturado 11 jul. 2013]. Disponível em: < http:/ / www.med.uzh.ch/Nachwuchsfoerderung/mentoring_report.pdf $>$

11. Pololi L, Knight S. Mentoring faculty in academic medicine: a new paradigm? Journal of General Internal Medicine 2005;20(9) [capturado 19 set. 2011]. Disponível em: < http://www.ncbi.nlm.nih.gov/pmc/articles/ PMC1490198/pdf/jgi_05007.pdf>.

12. Berk RA, Berg J, Mortimer R, Walton-Moss B, Yeo TP. Measuring the effectiveness of faculty mentoring relationships. Academic Medicine 2005; 80(1) [capturado 19 set. 2011]. Disponível em: http://ovidsp.tx.ovid.com/sp-.4.2a/ovidweb.cgi?T=JS\&PAGE $=$ fulltext $\& D=$ ovft $\& A N=00001888$ 200501000-00017\&NEWS $=$ N\&CSC $=$ Y \&CHANNEL $=$ Pub Med.

13. Jackson VA, Palepu A, Szalacha L, Caswell C, Carr PL, Inui T. "Having the right chemistry": a qualitative study of mentoring in academic medicine. Academic Medicine 2003;78(3) [capturado 19 set. 2011]. Disponível em: < http:/ / www.ncbi.nlm.nih.gov/pubmed/12634219\#>.

14. "empatia”, In. DICIONÁRIO Priberam da Língua Portuguesa [em linha], 2008-2013, [capturado 12 jan. 2012]. Disponível em: <http:/ / www.priberam.pt/DLPO/empatia>.

15. Davis OC, Nakamura J. A proposed model for an optimal mentoring environmental for medical residents: a literature review. Academic Medicine 2010;85(6) [capturado 19 set. 2011]. Disponível em: < http:/ / www.ncbi.nlm.nih. gov/pubmed/20505410>.

16. Bellodi PL. Mentors, students, and the undergraduate medical course: a virtuous circle. Revista bras educ med. 2011; 35(3) [capturado 19 set. 2011]. Disponível em: <http:/ / www.scielo.br/scielo.php?script=sci_arttext\&pi $\mathrm{d}=$ S0100-55022011000300012>.

17. Bussey-Jones J, Bernstein L, Higgins S, Malebranche D, Paranjape A, Genao I, Lee B, Branch W. Repaving the Road to Academic Success: The IMeRGE Approach to Peer Mentoring. Academic Medicine. 2006;81(7) [capturado 19 set. 2011]. Disponível em: < http://www.ncbi.nlm.nih.gov/ pubmed/16799297>.

18. Kalén S, Stenfors-Hayes T, Hylin U, Larm MF, Hindbeck $\mathrm{H}$, Ponzer S. Mentoring medical students during clinical 
courses: A way to enhance professional development. Medical Teacher 2010;32(8) [capturado 19 set. 2011]. Disponível em: <http:/ /informahealthcare.com/doi/abs/10.3109 /01421591003695295>.

19. Overeem K, Driessen EW, Arah OA, Lombarts KMJMH, Wollersheim HC, Ghrol RPTM. Peer mentoring in doctor performance assessment: strategies, obstacles and benefits. Medical Education. 2010;44(2) [4 abr. 2012]. Disponível em: http: / / www.mendeley.com/research/peer-mentoring-in-doctor-performance-assessment-strategies-obstacles-and-benefits/\#.

20. Bergstresser PR. Academic mentoring. Journal of Investigative Dermatology.2011;131(2) [capturado 19 set. 2011]. Disponível em: < http://www.nature.com/jid/journal/ v131/n2/full/jid2010396a.html>.

21. Bellodi PL, Chebabo R, Abensur SI, Martins MA. Mentoring: ir ou não ir, eis a questão: um estudo qualitativo. Revista bras educ med. 2011; 35(2) [capturado 19 set. 2011]. Disponível em: < http://www.scielo.br/scielo. php?script=sci_arttext\&pid=S0100-55022011000200013 >.

22. Stenfors-Hayes T, Kalén S, Hult H, Dahlgren L, Hindbeck $\mathrm{H}$, Ponzer S: Being a mentor for undergraduate medical students enhances personal and professional development. Med Teach 2010; 32 [capturado 11 jul. 2013]; 148-153. Disponível em: < http://informahealthcare.com/doi/ abs/10.3109/01421590903196995>.

23. Bellodi PL, Martins MA. Tutoria: mentoring na formação médica. 1 ed. São Paulo: Casa do Psicólogo; 2005.

24. Zerzan JT, Hess R, Schur E, Phillips RS, Rigotti N. Making the most of mentors: a guide for mentees. Academic Medicine 2009;84(1) [capturado 11 jul. 2013]; 140-144. Disponível em:< http://dgsomdiversity.ucla.edu/workfiles / lectures/Making\%20the\%20Most\%20of\%20Mentors.pdf $>$.

25. Rogers JF, Monteiro M, Nora A. Toward measuring the domains of mentoring. Family Medicine - Kansas City 2008;40(4) [capturado 11 jul. 2013]; 259. Disponível em:< http: / / www.stfm.org/fmhub/fm2008/April/John259. pdf>

26. Pololi LH, Knight SM, Dennis K., Frankel RM. Helping medical school faculty realize their dreams: an innovative, collaborative mentoring program. Academic Medicine 2002;77(5) [capturado 11 jul. 2013]; 377-384. Disponível em: http:/ /journals.lww.com/academicmedicine/Abstract/2002/05000/Helping_Medical_School_Faculty_Realize_Their.5.aspx.

27. Buddeberg-Fischer B, Herta KD. Formal mentoring programmes for medical students and doctors - a review of the Medline literature. Medical Teacher 2006; 28(3) [captu- rado 19 set. 2011]. Disponível em: < http://www.buddeberg-praxis.ch/_pdf/bb/Med_Teacher_2006.pdf>.

28. Ramanan RA, Taylor WC, Davis RB, Phillips RS. Mentoring Matters: Mentoring and Career Preparation in Internal Residency Training. Journal of General Internal Medicine 2006; 21(4) [capturado 4 abr. 2012]. Disponível em: <http:/ / www.ncbi.nlm.nih.gov/pubmed/16686809>.

29. Buddeberg-Fischer B, Stamm M, Buddeberg C. Academic career in medicine - requirements and conditions for successful advancement in Switzerland. BMC Health Services Research 2009;9(70) [capturado 19 set. 2011]. Disponível em: < http://www.biomedcentral.com/1472-6963/9/70>.

30. Colares MFA, Castro M, Peres CM, Passos ADC, Figueiredo JFC, Rodrigues MLV, Troncon LEA.. Group mentoring for junior medical students: perceptions of mentees and mentors. Revista bras educ med. 2009; 33(4) [capturado 19 set. 2011]. Disponível em:<http://www. scielo.br/scielo.php?script=sci_arttext\&pid=S0100$-55022009000400019 \& \operatorname{lng}=$ pt\&nrm=iso $>$.

31. Luckhaupt SE, Chin MH, Mangione CM, Phillips RS, Bell D, Leonard AC, Tsevat J. Mentorship in Academic General Internal Medicine: Results of a Survey of Mentors. Journal of General Internal Medicine 2005;20(11) [capturado 19 set. 2011]. Disponível em: <http://www.ncbi.nlm.nih.gov/ pubmed/16307626>.

32. Gusic ME, Zenni EA, Ludwig S, First LR. Strategies to design an effective mentoring program. The Journal of pediatrics 2010; 156(2) [capturado 11 jul. 2013]; 173-174. Disponível em:< http://www.jpeds.com/article/S00223476(09)01119-6/>.

33. Aguilar-da-Silva RH, Perim GL, Abdalla IG, Costa NMSC, Lampert JB, Stella RCR. Abordagens pedagógicas e tendências de mudanças nas escolas médicas. Revista bras educ med. 2009; 33(1supl.1) [capturado 19 set. 2011]; 53-62. Disponível em: <http://www. scielo.br/scielo.php?script=sci_arttext\&pid=S0100$-55022009000500006 \& \operatorname{lng}=$ en\&nrm=iso $>$.

34. Faculdade de Medicina de Marília. Boletim tutores. Outros programas de tutoria: a experiência da Faculdade de Medicina de Marília (FAMEMA), n.5; 2003.

35. Universidade Federal de Minas Gerais. Faculdade de Medicina. Notícias Internas. Tutoria completa 10 anos e seleciona novos professores; 2011 [capturado 19 set 2011]. Disponível em: < http://www.medicina.ufmg.br/ noticiasinternas $/ ? \mathrm{p}=4260>$.

36. Abensur SI, Bellodi PL. Tutores FMUSP. 2011 [capturado 19 set. 2011]. Disponível em: <http://www.fm.usp.br/ tutores $/>$. 


\section{CONTRIBUIÇÃO DOS AUTORES}

Todos os autores participaram na concepção, desenho, análise e interpretação dos dados do estudo, assim como da discussão e redação do texto final.

\section{CONFLITO DE INTERESSES}

Declarou não haver.

\section{ENDEREÇO PARA CORRESPONDÊNCIA}

Leandro Jerez Chaves

Rua Olegário Maciel, 482 - apto 305

Santa Helena - Juiz de Fora

CEP 36.015-350 - MG

E-mail: leandrojchavesufjf@gmail.com 